\title{
PROTEINS:
}

\section{The crystal structure of the catalytic domain of the Ser/Thr kinase PknA from M. tuberculosis shows an Src-like autoinhibited conformation}

\begin{tabular}{|r|l|}
\hline Journal: & PROTEINS: Structure, Function, and Bioinformatics \\
\hline Manuscript ID: & Prot-00517-2014.R1 \\
\hline Wiley - Manuscript type: & Structure Note \\
\hline Complete List of Authors: & $\begin{array}{l}\text { Wagner, Tristan; Max Planck Institute for Terrestrial Microbiology, - } \\
\text { Alexandre, Matthieu; Institut Pasteur, Structural Biology and Chemistry } \\
\text { Duran, Rosario; Institut Pasteur de Montevideo, Analytical Biochemistry } \\
\text { and Proteomics } \\
\text { Barilone, Nathalie; Institut Pasteur, Viral Neuroimmunology } \\
\text { Wehenkel, Annemarie; Institut Curie, Signalling, Neurobiology and Cancer } \\
\text { Alzari, Pedro; Institut Pasteur, CNRS URA 359, Unite de Biochimie } \\
\text { Structurale; } \\
\text { Bellinzoni, Marco; Institut Pasteur, Structural Biology and Chemistry }\end{array}$ \\
\hline Key Words: & $\begin{array}{l}\text { Mycobacterium tuberculosis, Ser/Thr phosphorylation, } \\
\text { Autophosphorylation, Autoinhibition, X-ray crystallography, Mass } \\
\text { spectrometry }\end{array}$ \\
\hline
\end{tabular}


The crystal structure of the catalytic domain of the Ser/Thr kinase PknA from M. tuberculosis shows an Src-like autoinhibited conformation

Tristan Wagner ${ }^{1,2,3 \S}$, Matthieu Alexandre ${ }^{1,2,3}$, Rosario Duran ${ }^{4,5}$, Nathalie Barilone $e^{1,2,3 \S}$, Annemarie Wehenkel ${ }^{1,2,3 \S}$, Pedro M. Alzari ${ }^{1,2,3}$, Marco Bellinzoni ${ }^{1,2,3^{*}}$

${ }^{1}$ Institut Pasteur, Unité de Microbiologie Structurale, 75724 Paris, France; ${ }^{2}$ CNRS UMR 3528, 75724 Paris, France; ${ }^{3}$ Université Paris Diderot, Sorbonne Paris Cité, Microbiologie Structurale, 75724 Paris, France; ${ }^{4}$ Unidad de Bioquímica y Proteómica Analíticas, Institut Pasteur de Montevideo, Uruguay; ${ }^{5}$ Unidad de Bioquímica y Proteómica Analíticas, Instituto de Investigaciones Biológicas Clemente Estable, Ministerio de Educación y Cultura, Uruguay

Short Title: Structure of the Ser/Thr protein kinase PknA

Keywords: Mycobacterium tuberculosis, Ser/Thr phosphorylation, autophosphorylation, autoinhibition, X-ray crystallography, mass spectrometry.

* Corresponding author: Marco Bellinzoni, marco.bellinzoni@ pasteur.fr

$\S$ Present address: TW: Max Planck Institute for Terrestrial Microbiology, Karl-vonFrisch-Straße, 35043 Marburg, Germany; NB: Institut Pasteur, Unité de NeuroImmunologie Virale, 75724 Paris cedex 15, France; AW: Institut Curie, 91405 Orsay, France 


\begin{abstract}
Signal transduction mediated by Ser/Thr phosphorylation in Mycobacterium tuberculosis has been intensively studied in the last years, as its genome harbors eleven genes coding for eukaryotic-like Ser/Thr kinases. Here we describe the crystal structure and the autophosphorylation sites of the catalytic domain of PknA, one of two protein kinases essential for pathogen's survival. The structure of the ligand-free kinase domain shows an auto-inhibited conformation similar to that observed in human Tyr kinases of the Src-family. These results reinforce the high conservation of structural hallmarks and regulation mechanisms between prokaryotic and eukaryotic protein kinases.
\end{abstract}


Originally thought to be restricted to the eukaryotic kingdom, reversible protein phosphorylation on serine, threonine and tyrosine residues has emerged as a widespread signaling mechanism in prokaryotes and has turned into a highly active field of research in modern molecular microbiology. Mycobacterium tuberculosis was among the first microorganisms for which genomic data indicated the presence of eukaryotic-like Ser/Thr kinases (STPKs) and has been intensively studied since, given the high interest in STPKs as possible targets for the development of new antituberculosis drugs ${ }^{1,2}$. PknA is found together with a second kinase, PknB, in a conserved operon that also includes two genes coding for FHA domain proteins (FhaA and FhaB), a penicillin-binding protein $(\mathrm{PbpA})$, a putative cell division protein (RodA), and the only known Ser/Thr phosphatase (PstP) of the M. tuberculosis genome ${ }^{1,2}$. The two kinases, PknA and $\mathrm{PknB}$, are thought to be involved in the control of cell division and were shown to be essential for mycobacterial growth ${ }^{2}$. Although PknB is one of the most studied eukaryotic-like bacterial kinases and the first for which the crystal structure became available ${ }^{1}$, the detailed signaling pathway(s) in which these STPKs could be involved remain elusive. Moreover, in contrast to $\mathrm{PknB}$, for which structural and biochemical evidence has started to shed light on its regulation mechanisms ${ }^{3}$, the absence of structural data for PknA has hampered further functional studies, despite the likely involvement of this kinase in common signaling pathways. We describe here the crystal structure of the catalytic domain of PknA and the identification of several autophosphorylation sites in the intracellular region, providing first clues about the regulation mechanism of this essential mycobacterial kinase.

\section{MATERIALS AND METHODS}

John Wiley \& Sons, Inc. 
Cloning, expression and purification. To produce recombinant soluble protein for structural studies, the portion of the $p k n A$ gene (rv0015c) coding for the predicted intracellular moiety $\left(\mathrm{PknA}_{1-336}\right)$ was amplified by $\mathrm{PCR}$ from the cosmid MTCY10H4, introducing a 5' DNA sequence coding for the TEV protease cleavage site (ENLYFQG), as well as a EcoRI restriction site at both ends. The purified PCR fragment was then cloned in the pET-28a vector leading to plasmid pM260, which was subsequently used to transform the E. coli strain BL21(DE3)pLysS for protein production. The transformed strain was grown at $30^{\circ} \mathrm{C}$ in $\mathrm{LB}$ medium supplemented with $50 \mu \mathrm{g} / \mathrm{ml}$ kanamycin and $30 \mu \mathrm{g} / \mathrm{ml}$ chloramphenicol, and protein overexpression was achieved by induction with $1 \mathrm{mM}$ IPTG once $\mathrm{OD}_{600} \sim 0.7$, followed by $3.0 \mathrm{~h}$ growth at $30^{\circ} \mathrm{C}$. Cells were then harvested by centrifugation, washed with PBS (140 $\left.\mathrm{mM} \mathrm{NaCl}, 2.7 \mathrm{mM} \mathrm{KCl}, 10 \mathrm{mM} \mathrm{Na} \mathrm{HPO}_{4}, 1.8 \mathrm{mM} \mathrm{KH}_{2} \mathrm{PO}_{4}, \mathrm{pH} 7.3\right)$ and frozen at $80^{\circ} \mathrm{C}$. Pellets were thawed, resuspended in lysis buffer $\left(50 \mathrm{mM} \mathrm{NaH}_{2} \mathrm{PO}_{4}, 500 \mathrm{mM}\right.$ $\mathrm{NaCl}, 25 \mathrm{mM}$ imidazole, 5\% glycerol; $\mathrm{pH}$ 8.0) and lysed by sonication on ice; lysates were then centrifuged at $26,800 \times g$ for $1 \mathrm{~h}$, filtered on a $0.45 \mu \mathrm{m}$ membrane and loaded with a peristaltic pump onto a $1 \mathrm{ml}$ HisTrap $\mathrm{Ni}^{2+}$-IMAC column (GE Healthcare). $\mathrm{PknA}_{1-336}$ was eluted applying a $25-400 \mathrm{mM}$ imidazole gradient in the same buffer; the fractions containing the recombinant protein, as confirmed by $12 \%$ SDS-PAGE, were pooled and dialysed overnight at $18^{\circ} \mathrm{C}$ against a specific PknA buffer (25 mM Hepes-Na pH 8.0, $500 \mathrm{mM} \mathrm{NaCl}, 5 \%$ glycerol, $1 \mathrm{mM}$ DTT). During the dialysis a recombinant His $_{6}$-tagged TEV protease was added to a 1:35 w/w ratio. The dialyzed sample was passed by gravity flow through $1.0 \mathrm{ml}$ of Ni-NTA resin (Qiagen) in order to eliminate the TEV protease and the cleaved $\mathrm{His}_{6}$ expression tag, eluted with $3 \mathrm{ml}$ of PknA buffer supplemented with $50 \mathrm{mM}$ imidazole, concentrated and injected onto a HiLoad 16/60 Superdex 75 size exclusion column (GE 
Healthcare), equilibrated in the PknA buffer (with no imidazole) and run at $1 \mathrm{ml} / \mathrm{min}$. Peak fractions containing $\mathrm{PknA}_{1-336}$ were pooled and concentrated up to $36 \mathrm{mg} / \mathrm{ml}$ with a $10 \mathrm{kDa}$-cut off Vivaspin concentrator (Sartorius). The concentrated protein, with a purity $>95 \%$ as estimated by Coomassie blue staining on SDS-PAGE, was flash-frozen in liquid nitrogen and stored at $-80^{\circ} \mathrm{C}$.

Phosphorylation site identification by tandem mass spectrometry. Protein samples were digested with different proteolytic enzymes (chymotrypsin, trypsin and endoproteinase $\mathrm{GluC}$ ) by incubation overnight at $37^{\circ} \mathrm{C}$. The resulting peptides were separated using a nano-HPLC system (Proxeon EasynLC, Thermo) fitted with a reverse-phase column (easy C18 column, $3 \mu \mathrm{m} ; 75 \mu \mathrm{m}$ ID $\times 10 \mathrm{~cm}$; Proxeon, Thermo) eluted with a $0.1 \%(\mathrm{v} / \mathrm{v})$ formic acid in a water to acetonitrile gradient (0-35\% acetonitrile in $40 \mathrm{~min}$; flow $400 \mathrm{nl} / \mathrm{min}$ ). Online MS analysis was carried out in the LTQ Velos nano-ESI-linear ion trap instrument (Thermo) in data dependent acquisition mode (full scan followed by MS/MS of the top 5 peaks in each segment, using a dynamic exclusion list). Peptide sequences were assigned using Mascot and Sequest search engines. An in-house Mascot v.2.0 version was used for searching the Swissprot database (20121102) with the following parameters: taxonomy: Mycobacterium tuberculosis complex; peptide tolerance: 1.5 Da; MS/MS tolerance: 0.8 Da; methionine oxidation, and Ser/Thr phosphorylation as the allowed variable modifications. The data was filtered using significance threshold $\mathrm{p}<0.05$ and ion score $>30$. In addition raw MS/MS spectra were interpreted with the Proteome Discoverer software package (v.1.3.0.339, Thermo). We considered positive phosphosite identification when more than one spectra for the phosphopeptide was 
obtained, pRS probability was $>95 \%$ and manual inspection of the MS/MS spectra showed at least two confirmatory fragment ions.

Crystallization. Initial crystallization screenings of $\mathrm{PknA}_{1-336}$ were carried out at $18^{\circ} \mathrm{C}$ by the sitting drop method in a 96-well format $(200+200 \mathrm{nl}$ drops dispensed by a Cartesian nanolitre system). Microcrystals were identified in a crystallization condition containing 4.3 M NaCl, $100 \mathrm{mM}$ Hepes-Na pH 8.0, which was refined in several steps by preparing hand-made hanging drops in 24-well plates at the same temperature. The final crystallization buffer was $3.8 \mathrm{M} \mathrm{NaCl}, 100 \mathrm{mM}$ MES pH 6.7, $14.3 \mathrm{mM} \mathrm{Na}$ acetate $\mathrm{pH} 4.5$, with which crystals suitable to structural determination grew in 8 to 10 days by microseeding from the original condition. Crystals were flashfrozen in liquid nitrogen after quick soaking in a cryoprotectant mixture made of $75 \%$ crystallization solution and $25 \%$ glycerol.

Data collection, structure determination and refinement. X-ray diffraction data were collected at $100 \mathrm{~K}$ on the beamline ID14-2 at the European Synchrotron Radiation Facility (ESRF) (Grenoble, France). The structure was solved by molecular replacement with Phaser ${ }^{4}$, using the split N-terminal (residues 5-97) and C-terminal (residues 99-276) domains of the STPK PknB from M. tuberculosis (pdb entry 1o6y; ${ }^{5}$ ) as separate search models. The molecular replacement solution, composed of three kinase domains per asymmetric unit, was refined with either refmac5 ${ }^{6}$ or autoBUSTER, applying local structure similarity restraints for NCS ${ }^{7}$. The model was validated through the Molprobity server ${ }^{8}$. Figures were generated and rendered with PyMOL (The PyMOL Molecular Graphics System, Version 1.5, Schrödinger, LLC). 
Coordinates and structure factors have been deposited in the pdb under the accession code $4 \mathrm{X} 3 \mathrm{~F}$.

\section{RESULTS AND DISCUSSION}

Overall structure. Although attempts to overexpress the N-terminal catalytic domain (residues 1-276) of PknA in E. coli were unsuccessful, we were able to express and purify the whole intracellular moiety of the kinase, including the entire juxtamembrane segment (residues 277-336), as a His ${ }_{6}$-tagged soluble protein to a satisfactorily yield ( $3.5 \mathrm{mg} / \mathrm{l}$ of culture). This construct is active on the FHA domain substrate GarA, as reported ${ }^{9}$. After proteolytic removal of the affinity tag, the recombinant protein crystallized in high salt conditions (around $4 \mathrm{M} \mathrm{NaCl}$ ). The crystals belong to the monoclinic space group $\mathrm{C} 2$ and the $3 \mathrm{D}$ structure has been determined to $2.9 \AA$ using molecular replacement methods (Table 1). The asymmetric unit contains three molecules of PknA, all of which were traceable from the Nterminus (with the exception of Met1 and Ser2, not visible in all chains) to residue Pro289, corresponding to the whole catalytic domain plus the first 13 residues of the juxtamembrane segment. In contrast, no supporting electron density was observed for the remaining part of the juxtamembrane segment (residues Gly290 to Gly336). The catalytic domain shows the canonical, bi-lobal protein kinase fold, with an $\mathrm{N}$-terminal lobe (Met1 to Val98), composed of a five-stranded antiparallel $\beta$-sheet plus the functionally important $\alpha \mathrm{C}$ helix (Pro52 to Ala66), followed by a mostly $\alpha$-helical Cterminal lobe (residues Asn98 to Gly277) that includes helices $\alpha \mathrm{D}$ to $\alpha \mathrm{I}$ (Fig. 1A). Just outside the catalytic domain, the N-terminal visible portion of the juxtamembrane segment makes a left-handed PPII helix extension to the C-terminal lobe of the kinase (Fig. 1B). This segment makes two H-bonds with the catalytic domain, one between 
the amide nitrogen of Arg278 and the carbonyl oxygen of Ala273 (at the end of the helix $\alpha \mathrm{I}$ ), the other between the carbonyl of Pro281 and the hydroxyl of Tyr 261 in the $\alpha \mathrm{G}$ to $\alpha \mathrm{I}$ linker. In contrast to earlier reports ${ }^{10}$, the mostly disordered juxtamembrane region of PknA suggests that this region might be dispensable for enzymatic activity. In agreement with this hypothesis, we observed that a shorter PknA construct lacking most of the juxtamembrane segment, $\mathrm{PknA}_{1-290}$, was indeed capable of phosphorylating the substrate GarA (data not shown).

Despite our attempts to get co-crystals of PknA with commercially available nonhydrolysable ATP analogs, the catalytic site, situated at the interface between the two lobes as in all Ser/Thr and Tyr protein kinases, shows no bound nucleotide or divalent ion. Nevertheless, the ensemble of well-documented elements of Hanks-type kinases is easily identifiable. The activation loop is comprised of 28 residues between the sequence motifs DFG (Asp159-Phe160-Gly161) and APE (Ala185-Pro186-Glu187), two of the extremely conserved features of protein kinases (Fig. 1C; Fig. 2A). Among the other traits of Hanks-type kinases, the conserved lysine on the $\beta 3$ strand (Lys42), involved in the binding of the ATP $\alpha$ and $\beta$ phosphates, the equally conserved glutamate on the critical $\alpha \mathrm{C}$ helix (Glu61), involved in the correct positioning of the same lysine by a salt bridge, and the so-called HRD motif (His139-Arg140-Asp141) that identifies the catalytic loop and allow to classify PknA as an RD-kinase (Fig. 2A). However, the whole activation loop and part of the following P+1 loop are disordered in each of the three chains in the asymmetric unit, as judged by the lack of supporting electron density for residues 168-177 (chain A), 164-174 (chain B), 164175 (chain C). 
PknA autophosphorylation. The autophosphorylation pattern of PknA was analyzed by mass spectrometry. The protein shows a heterogenous phosphorylation pattern with multiple sites identified in good agreement with previous results for PknA and other mycobacterial Ser/Thr protein kinases ${ }^{11,12}$. MS/MS analysis of the PknA tryptic digest allowed to detect 21 phosphorylated peptides and to identify 18 phosphorylation sites (Supplementary Table 1). For two phosphopetides (sequence 292-304 and 279-291), the phosphoresidue location could not be unambiguously determined (Supplementary Table 1). However, no tryptic peptides corresponding to the activation loop sequence could be identified. To increase sequence coverage and improve phosphosite identification, we digested PknA with different proteolytic enzymes. Both chymotryptic and endoproteinase GluC digests confirmed some of the previously identified phosphoresidues (data not shown) and led to further identification of new sites (Supplementary Table 1). In particular, a triply charged ion ( $m / z$ 1441.71) could be assigned to a peptide (residues 160-199) containing four phosphorylated residues and one methionine oxidation with a significant Mascot ion score (experimental $\mathrm{Mr}=4322.11$, calculated $\mathrm{Mr}=4321.78)$. This segment, which overlaps with the kinase activation loop, includes the highly conserved Thr residues whose phosphorylation was important for activation in other mycobacterial Ser/Thr protein kinases ${ }^{12}$. However, the fragmentation properties of this multiply phosphorylated peptide preclude the detection of sequence-specific ion signals, thus rendering the localization of phosphorylated sites difficult.

In a previous report, eight phosphorylation sites were identified in $\mathrm{PknA}_{1-338}{ }^{11}$. Except for Ser212, all the other sites were also detected in our experimental conditions. In addition we report 12 new phosphorylation sites and provide evidence 
supporting the presence of multiple phosphorylation sites in the activation loop, although the exact sites could not be unambiguously assigned. It should be noted that only four of these phosphoresidues (Thr21, Ser46, Thr152, Thr224) were visible in the electron density maps of at least one PknA molecule, but they are highly exposed to solvent and possibly do not play a regulatory or structural role.

PknA displays an autoinhibited state. The relative position of the two lobes suggests that PknA has been crystallized in a closed state, as observed for PknB in complex with nucleotides or for the ternary complex of human PKA (pdb entry 1atp) (Fig. 2B), generally considered as the prototype of the active form of Ser/Thr kinases ${ }^{13}$. The plasticity of the activation loop and lack of supporting electron density is similar to what is observed for the active conformation of the related M. tuberculosis Ser/Thr kinase $\mathrm{PknB}$, as reported independently by different teams ${ }^{2}$. However, the conformation of PknA corresponds to an inactive state, close to the autoinhibited structure of the kinase domain of Staphylococcus aureus PknB (Fig. 2B) (pdb entry 4eqm; ${ }^{14}$ ). Furthermore, the N-terminus of PknA helix $\alpha \mathrm{C}$ is rotated approximately $17^{\circ}$ outwards of the active site pocket with respect to the same helix in $\mathrm{PknB}$, hampering formation of the salt bridge Glu61 - Lys42 that is a hallmark of active protein kinases (Fig. 2B). A similar, but less pronounced tilt of $\alpha \mathrm{C}$ was also observed in the inactive conformation of mycobacterial PknE (pdb entry 2h34) (Fig. 2B).

Noteworthy, the conformation of apo PknA represents an example of the wellcharacterized CDK/Src-like inactive conformation, observed in a number of structures of both Ser/Thr and Tyr kinases (reviewed in ${ }^{15}$ ). The known features of this autoinhibited conformation include the closure of the lobes and the swinging out of 
$\alpha \mathrm{C}$. Furthermore, the N-terminal portion of the activation loop (residues Ile162 to Asp167 in PknA), which immediately follows the DFG motif, assumes a well defined one-turn helical conformation that packs against $\alpha \mathrm{C}$, stabilizing its outward orientation and creating a barrier to the Lys-Glu salt bridge (Fig. 2A). The presence of such an inhibitory helix (or A-loop helix for activation loop) was first observed in the crystal structures of the human tyrosine kinases c-Src in complex with AMP-PNP (pdb entry $2 \mathrm{src} ;{ }^{16}$ ), as well as Hrc in complex with the pyrazolo-pyrimidine inhibitor PP1 (pdb entry 1qcf; ${ }^{17}$ ). In chains B and C of PknA, however, a slightly different autoinhibited conformation is observed, with the traceable residues of the activation loop, despite not forming an $\alpha$-helix, still hindering the Lys-Glu interaction and the carboxylic group of Glu61 positioned instead to form a salt bridge with the guanidinium of Arg140, part of the HRD motif and adjacent to the strictly conserved Asp141 within the catalytic loop. This interaction, that contributes to stabilizing the $\alpha \mathrm{C}$ outward orientation, was also observed in the two tyrosine kinases mentioned above and has recently been proposed to represent an intermediate state through the path to the active conformation ${ }^{15}$. Consistently with the present crystal structure of PknA representing an inactive conformation, the catalytic and regulatory spines (two conserved intramolecular networks between the $\mathrm{N}$ - and C-lobes in active protein kinases ${ }^{18}$ ) are also not properly assembled.

A conserved activation mechanism in mycobacterial kinases? The crystal structures of the catalytic domains of PknB and PknE, the two other M. tuberculosis predicted receptor kinases for which a three-dimensional structure of the kinase domain is available, showed in both cases an homodimeric form referred to as 'back-to-back', since the dimerization interface involves the $\mathrm{N}$-terminal lobe surface located opposite 
to the active site ${ }^{2}$. This 'back-to-back' dimerization has been proposed to act as a general allosteric activation mechanism in receptor-like mycobacterial kinases ${ }^{3}$, in which homodimer formation is required in order to achieve activation loop phosphorylation and kinase activation. A similar mechanism might also be operational in PknA, since the equivalent $\mathrm{N}$-terminal homodimerization interface is well conserved (Supplementary Figure 1) and the activation loop has several phosphorylation sites. However, further experimental evidence is necessary to validate this model or, alternatively, support the recently postulated existence of a hierarchical kinase network in M. tuberculosis, according to which phosphorylation by $\mathrm{PknB}$ would be required for PknA activation ${ }^{9}$.

\section{ACKNOWLEDGEMENTS}

We are grateful to Clément Moroldo for generating and testing constructs for recombinant protein expression, to Ahmed Haouz and Patrick Weber for robot-driven crystallization screenings, and to Gwénaëlle André-Leroux and María-Natalia Lisa for many helpful discussions. The expression plasmid for the TEV protease was a kind gift from Helena Berglund (Karolinska Institute, Stockholm, Sweden). We also acknowledge the ESRF (Grenoble, France) for granting access to its macromolecular crystallography facilities. This work has been funded by grants from the Institut Pasteur, the CNRS and the European Commission (contract number LSHP-CT-2005018923).

\section{REFERENCES}

1. Wehenkel A, Bellinzoni M, Graña M, Duran R, Villarino A, Fernandez P, Andre-Leroux G, England P, Takiff H, Cerveñansky C, Cole ST, Alzari PM. 
Mycobacterial Ser/Thr protein kinases and phosphatases: physiological roles and therapeutic potential. Biochim Biophys Acta 2008;1784:193-202.

2. Prisic S, Husson RN. Mycobacterium tuberculosis Serine/Threonine Protein Kinases. Microbiol Spectr 2014;2.

3. Lombana TN, Echols N, Good MC, Thomsen ND, Ng H-L, Greenstein AE, Falick AM, King DS, Alber T. Allosteric activation mechanism of the Mycobacterium tuberculosis receptor Ser/Thr protein kinase, PknB. Structure 2010;18:1667-1677.

4. McCoy AJ, Grosse-Kunstleve RW, Adams PD, Winn MD, Storoni LC, Read RJ. Phaser crystallographic software. J Appl Crystallogr 2007;40:658-674.

5. Ortiz-Lombardía M, Pompeo F, Boitel B, Alzari PM. Crystal structure of the catalytic domain of the PknB serine/threonine kinase from Mycobacterium tuberculosis. J Biol Chem 2003;278:13094-13100.

6. Murshudov GN, Skubak P, Lebedev AA, Pannu NS, Steiner RA, Nicholls RA, Winn MD, Long F, Vagin AA. REFMAC5 for the refinement of macromolecular crystal structures. Acta Crystallogr D Biol Crystallogr 2011;67:355-367.

7. Smart OS, Womack TO, Flensburg C, Keller P, Paciorek W, Sharff A, Vonrhein C, Bricogne G. Exploiting structure similarity in refinement: automated NCS and target-structure restraints in BUSTER. Acta Crystallogr D Biol Crystallogr 2012;68:368-380.

8. Chen VB, Arendall WB3, Headd JJ, Keedy DA, Immormino RM, Kapral GJ, Murray LW, Richardson JS, Richardson DC. MolProbity: all-atom structure validation for macromolecular crystallography. Acta Crystallogr D Biol Crystallogr 2010;66:12-21. 
9. Baer CE, Iavarone AT, Alber T, Sassetti CM. Biochemical and Spatial Coincidence in the Provisional Ser/Thr Protein Kinase Interaction Network of Mycobacterium tuberculosis. J Biol Chem 2014;289:20422-20433.

10. Thakur M, Chaba R, Mondal AK, Chakraborti PK. Interdomain interaction reconstitutes the functionality of PknA, a eukaryotic type Ser/Thr kinase from Mycobacterium tuberculosis. J Biol Chem 2008;283:8023-8033.

11. Molle V, Zanella-Cleon I, Robin J-P, Mallejac S, Cozzone AJ, Becchi M. Characterization of the phosphorylation sites of Mycobacterium tuberculosis serine/threonine protein kinases, $\mathrm{PknA}, \mathrm{PknD}, \mathrm{PknE}$, and $\mathrm{PknH}$ by mass spectrometry. Proteomics 2006;6:3754-3766.

12. Duran R, Villarino A, Bellinzoni M, Wehenkel A, Fernandez P, Boitel B, Cole ST, Alzari PM, Cerveñansky C. Conserved autophosphorylation pattern in activation loops and juxtamembrane regions of Mycobacterium tuberculosis Ser/Thr protein kinases. Biochem Biophys Res Commun 2005;333:858-867.

13. Knighton DR, Zheng JH, Eyck Ten LF, Ashford VA, Xuong NH, Taylor SS, Sowadski JM. Crystal structure of the catalytic subunit of cyclic adenosine monophosphate-dependent protein kinase. Science 1991;253:407-414.

14. Rakette S, Donat S, Ohlsen K, Stehle T. Structural Analysis of Staphylococcus aureus Serine/Threonine Kinase PknB. PLoS One 2012;7:e39136.

15. Jura N, Zhang X, Endres NF, Seeliger MA, Schindler T, Kuriyan J. Catalytic Control in the EGF Receptor and Its Connection to General Kinase Regulatory Mechanisms. Mol Cell 2011;42:9-22.

16. Xu W, Doshi A, Lei M, Eck MJ, Harrison SC. Crystal structures of c-Src reveal features of its autoinhibitory mechanism. Mol Cell 1999;3:629-638.

17. Schindler T, Sicheri F, Pico A, Gazit A, Levitzki A, Kuriyan J. Crystal John Wiley \& Sons, Inc. 
structure of Hck in complex with a Src family-selective tyrosine kinase inhibitor. Mol Cell 1999;3:639-648.

18. Kornev AP, Taylor SS. Defining the conserved internal architecture of a protein kinase. Biochim Biophys Acta 2010;1804:440-444.

19. Robert X, Gouet P. Deciphering key features in protein structures with the new ENDscript server. Nucleic Acids Res 2014;42:W320-W324.

20. Gay LM, Ng H-L, Alber T. A conserved dimer and global conformational changes in the structure of apo-PknE Ser/Thr protein kinase from Mycobacterium tuberculosis. J Mol Biol 2006;360:409-420.

John Wiley \& Sons, Inc. 


\section{FIGURE LEGENDS}

Fig. 1. (A, B) Cartoon representation of the overall structure of the catalytic domain of PknA in unliganded form; secondary structure elements are indicated. The modeled portion of the juxtamembrane segment, which folds as a left-handed PPII helix, is shown in orange. The phosphorylated residues identified in electron density maps are indicated. (C) Sequence alignment of the N-terminal catalytic domains of the transmembrane receptor M. tuberculosis kinases PknA, PknB, PknD, PknE, PknF, PknH, PknI, PknJ, PknL that all share the same domain topology ${ }^{1,2,9}$; PknG and PknK, which are soluble kinases, are omitted. The secondary structure elements of the catalytic domain of PknA (this work) have been depicted through ESPript 3.0 ${ }^{19}$.

Fig. 2. (A) Close view of the active site pocket in PknA. The activation segment is highlighted in orange, and key conserved residue motifs known to be involved in the phosphorylation process or in structuring a catalytically competent kinase are shown in sticks, i.e. the motifs HRD (His139 to Asp141), DFG (Asp159 to Gly161), APE (Ala185 to Glu187). The N-terminal modeled portion of the activation loop, which is structured as a one-turn inhibitory $\alpha$-helix packing against $\alpha \mathrm{C}$, is shown in red. The unassembled catalytic and regulatory spines (C-spine in light brown, R-spine in yellow) are shown with a semitransparent surface representation. See the text for details. (B) Lateral view of the superimposed catalytic domains of PknA (yellow, this study), M. tuberculosis PknB in complex with AMP-PCP (pdb entry 1o6y, light brown; ${ }^{5}$ ), M. tuberculosis PknE (green; pdb entry $2 \mathrm{~h} 34,{ }^{20}$ ), S. aureus PknB (gray; pdb entry 4 eqm,${ }^{14}$ ) and the human cAMP-dependent kinase PKA in ternary complex with $\mathrm{MgATP}$ and an inhibitory peptide, shown as the paradigm of the active conformation of a Hanks-type protein kinase (purple; pdb entry 1atp, ${ }^{13}$ ). The dashed 


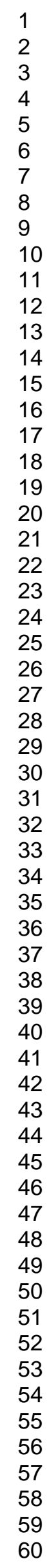

lines indicate the different orientation of the $\alpha \mathrm{C}$ helix axis when comparing PknA (yellow) and PknB (light brown).

John Wiley \& Sons, Inc. 
Table 1. Crystallographic data collection and refinement statistics. Values relative to the highest resolution shell are within parentheses.

\begin{tabular}{|l|c|}
\hline & $\begin{array}{c}\text { His }_{6} \text {-tagged SeMet- } \\
\text { labelled, } \\
\text { centred monoclinic }\end{array}$ \\
\hline Data collection & ESRF ID14-2 \\
\hline Synchrotron beamline & 0.933 \\
\hline Wavelength $(\AA)$ & $\mathrm{C} 2$ \\
\hline Space group & $102.31,150.18,96.54$ \\
\hline Cell dimensions $a, b, c(\AA)$ & $90.0,96.98,90.0$ \\
\hline$\alpha, \beta, \gamma\left({ }^{\circ}\right)$ & $43.57-2.90$ \\
\hline Resolution $(\AA)$ & $(3.06-2.90)$ \\
\hline$R_{\text {merge }}$ & $0.107(1.197)$ \\
\hline$I / \sigma(I)$ & $8.3(1.1)$ \\
\hline CC $(1 / 2)(\%)$ & $99.5(47.1)$ \\
\hline Unique reflections & 31961 \\
\hline Completeness $(\%)$ & $99.6(99.1)$ \\
\hline Redundancy & $3.6(3.5)$ \\
\hline & \\
\hline Refinement & \\
\hline Resolution $(\AA)$ & $37.98-2.90$ \\
\hline No. reflections* & 30353 \\
\hline$R_{\text {work }} R_{\text {free }}(\%)$ & $21.9 / 24.3$ \\
\hline Average B-factors $\left(\AA^{2}\right)$ & 95.1 \\
\hline Macromolecule & 95.1 \\
\hline Solvent & 54.7 \\
\hline R.m.s deviations & 0.010 \\
\hline Bond lengths $(\AA)$ & 1.289 \\
\hline Bond angles $\left({ }^{\circ}\right)$ & $4 X 3 \mathrm{~F}$ \\
\hline PDB \# & \\
\hline$*$ Excluding the $\mathrm{R}_{\text {free }}$ set $(5 \%$ of total reflections). \\
\hline
\end{tabular}



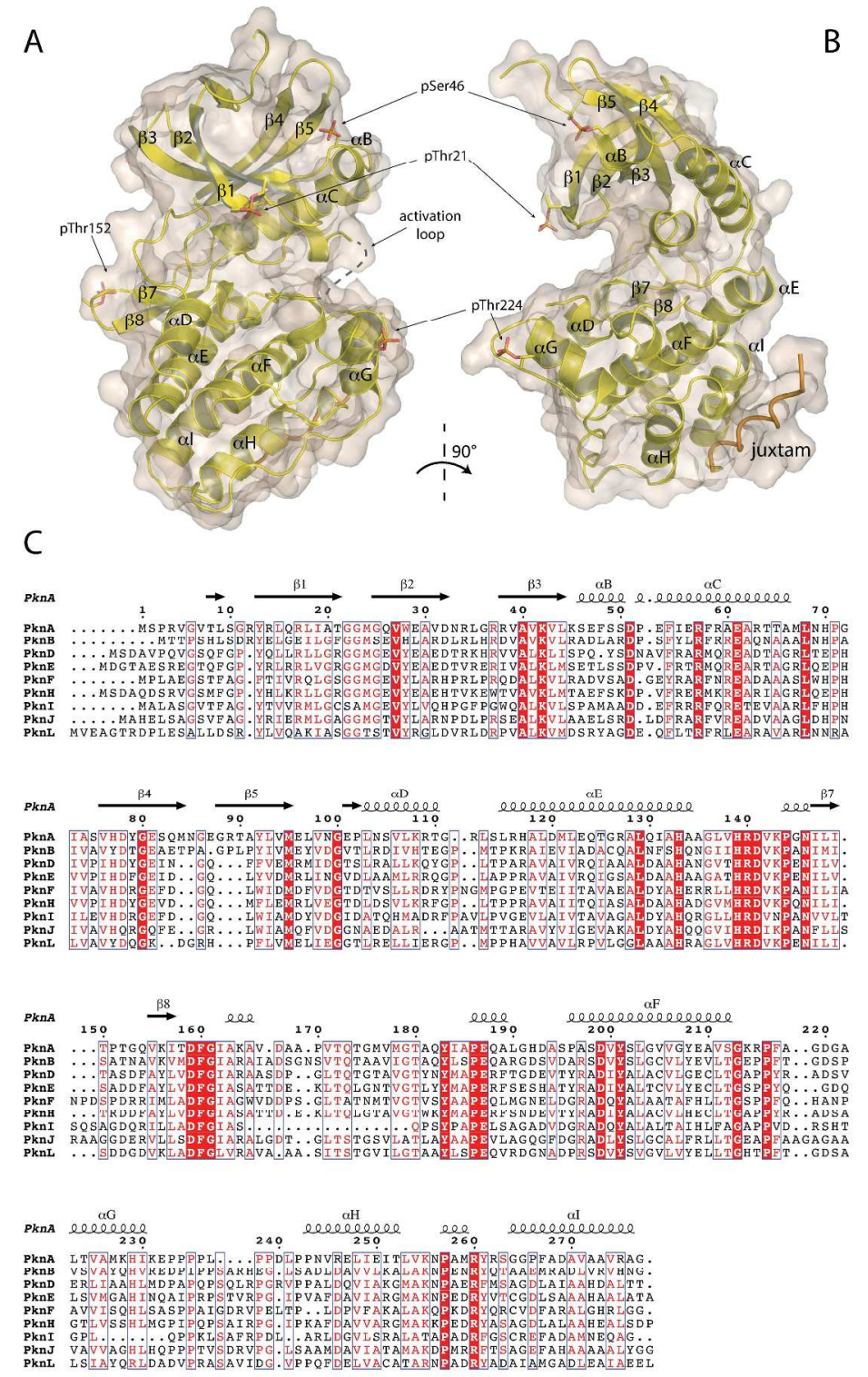

Fig. 1. (A, B) Cartoon representation of the overall structure of the catalytic domain of PknA in unliganded form; secondary structure elements are indicated. The modeled portion of the juxtamembrane segment, which folds as a left-handed PPII helix, is shown in orange. The phosphorylated residues identified in electron density maps are indicated. (C) Sequence alignment of the $\mathrm{N}$-terminal catalytic domains of the transmembrane receptor M. tuberculosis kinases PknA, PknB, PknD, PknE, PknF, PknH, PknI, PknJ, PknL that all share the same domain topology ${ }^{3,19}$; PknG and PknK, which are soluble kinases, are omitted. The secondary structure elements of the catalytic domain of PknA (this work) have been depicted through ESPript $3.0^{32}$.

$266 \times 436 \mathrm{~mm}(300 \times 300$ DPI $)$ 

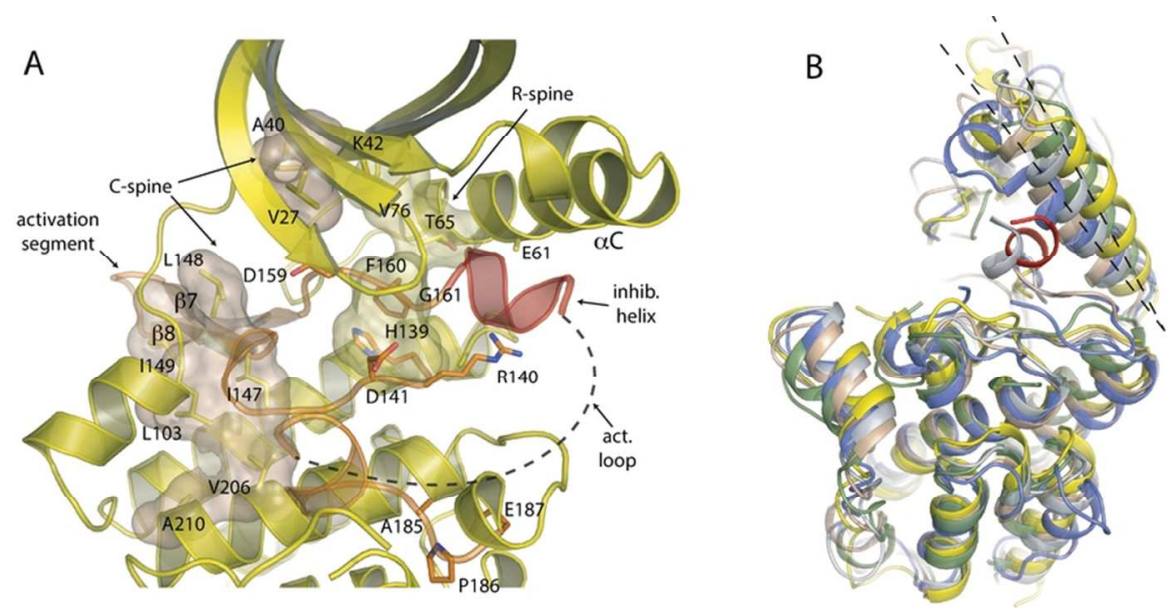

Fig. 2. (A) Close view of the active site pocket in PknA. The activation segment is highlighted in orange, and key conserved residue motifs known to be involved in the phosphorylation process or in structuring a catalytically competent kinase are shown in sticks, i.e. the motifs HRD (His139 to Asp141), DFG (Asp159 to Gly161), APE (Ala185 to Glu187). The N-terminal modeled portion of the activation loop, which is structured as a one-turn inhibitory a-helix packing against $\mathrm{aC}$, is shown in red. The unassembled catalytic and regulatory spines ( $\mathrm{C}$-spine in light brown, $\mathrm{R}$-spine in yellow) are shown with a semitransparent surface representation. See the text for details. (B) Lateral view of the superimposed catalytic domains of PknA (yellow, this study), M. tuberculosis PknB in complex with AMP-PCP (pdb entry 106y, light brown; ${ }^{9}$ ), $M$. tuberculosis PknE (green; pdb entry 2h34, ${ }^{31}$ ), S. aureus PknB (gray; pdb entry 4eqm, ${ }^{26}$ ) and the human CAMP-dependent kinase PKA in ternary complex with MgATP and an inhibitory peptide, shown as the paradigm of the active conformation of a Hanks-type protein kinase (purple; pdb entry 1 atp, ${ }^{24}$ ). The dashed lines indicate the different orientation of the aC helix axis when comparing PknA (yellow) and PknB (light brown).

$84 \times 39 \mathrm{~mm}(300 \times 300 \mathrm{DPI})$ 